\title{
Development of Diode Based High Energy X-ray Spatial Dose Distribution Measuring Device
}

\author{
Jeonghee Lee', Ikhyun Kim', Jong-Won Park', Yong-Kon Lim', Myungkook Moon², Sangheon Lee ${ }^{3}$, Chang Hwy Lim ${ }^{1, *}$ \\ ${ }^{1}$ Korea Research Institute of Ships \& Ocean Engineering, Daejeon, Korea; ${ }^{2}$ Korea Atomic Energy Research Institute, Daejeon, Korea; ${ }^{3}$ Daon Technology Co., \\ Ltd., Deajeon, Korea
}

\section{Original Research}

Received June 7, 2018

Revision August 23, 2018

Accepted September 12, 2018

Corresponding author: Chang Hwy Lim

Korea Research Institute of Ships \& Ocean Engineering, 32 Yuseong-daero 1312 beon-gil, Yuseong-gu, Deajeon 34103, Korea

Tel: +82-42-866-3855

Fax: +82-42-866-3819

E-mail:hwy77@kriso.re.kr

This is an Open-Access article distributed under the terms of the Creative Commons Attribution NonCommercial License (http://creativecommons.org/ licenses/by-nc/4.0) which permits unrestricted noncommercial use, distribution, and reproduction in any medium, provided the original work is properly cited.

Copyright $\odot 2018$ The Korean Association for Radiation Protection

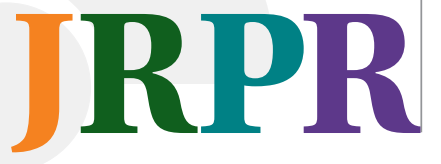

Background: A cargo container scanner using a high-energy X-ray generates a fan beam X-ray to acquire a transmitted image. Because the generated X-rays by LINAC may affect the image quality and radiation protection of the system, it is necessary to acquire accurate information about the generated X-ray beam distribution. In this paper, a diode-based multi-channel spatial dose measuring device for measuring the X-ray dose distribution developed for measuring the high energy X-ray beam distribution of the container scanner is described.

Materials and Methods: The developed high-energy X-ray spatial dose distribution measuring device can measure the spatial distribution of X-rays using 128 diode-based X-ray sensors. And precise measurement of the beam distribution is possible through automatic positioning in the vertical and horizontal directions. The response characteristics of the measurement system were evaluated by comparing the signal gain difference of each pixel, response linearity according to $\mathrm{X}$-ray incident dose change, evaluation of resolution, and measurement of two-dimensional spatial beam distribution.

Results and Discussion: As a result, it was found that the difference between the maximum value and the minimum value of the response signal according to the incident position showed a difference of about $10 \%$, and the response signal was linearly increased. And it has been confirmed that high-resolution and two-dimensional measurements are possible.

Conclusion: The developed X-ray spatial dose measuring device was evaluated as suitable for dose measurement of high energy X-ray through confirmation of linearity of response signal, spatial uniformity, high resolution measuring ability and ability to measure spatial dose. We will perform precise measurement of the X-ray beamline in the container scanning system using the $\mathrm{X}$-ray spatial dose distribution measuring device developed through this research.

Keywords: Container scanner, High energy X-ray, X-ray spatial dose distribution, Diode array

\section{Introduction}

Container scanner for non-destructive test for internal detects in harbor container vehicles use high-energy $\mathrm{X}$-rays in $\mathrm{MeV}$ and generally use linear array detectors to scan large containers efficiently. In addition, container scanner using X-rays should be able to acquire high-quality images for accurate inspection of objects inside containers. To this end, high-dose, high-energy X-rays should be used [1, 2]. In order to acquire high quality internal images of containers and to minimize the outside leakage of radiation, the X-rays of the container scanner must be irradiated only in a necessary direction. To this end, a collimator for concentrating X-rays and a large shielding structure is re- 
quired [3]. The collimator should be able to precisely align to the angle of X-ray beam and adjust the beam width so that the X-ray can be accurately incident on the pixels of the image detector. In addition, it is necessary to precisely verify the adjustment of X-ray beam angle and beam width using the collimator. If the exact spatial distribution of the X-ray is not measured, the X-ray beam width may become larger or smaller than necessary, which may result in scattered X-rays or a reduction in the number of photons reaching the detector causing deterioration of image quality. It may also cause difficulties in performing accurate alignment of the collimators between the X-ray generator and the detector. Therefore, it is necessary to verify the exact information on the $\mathrm{X}$-ray beams by measuring the $\mathrm{X}$-ray spatial distribution. For this purpose, the available sensors can be constructed by using ion chambers, diodes, plastic scintillators, etc. $[4,5]$. The ion chamber is widely used as a general X-ray dose measuring device because it can measure the absolute value of the dose and have the linearity of the response signal. Since, however, it uses the average value of the measurement space, the blurring phenomenon of the distribution image may occur at the position where the X-ray distribution suddenly changes in the measurement of the spatial distribution. In addition, since the gas is used for the $\mathrm{X}$-ray reaction, the reaction efficiency to high energy $\mathrm{X}$-rays is low so this method generates a very low current for small pixels. As a result, it may be difficult to obtain signals due to internal and external noises. If this method is implemented with an array, it must include circuitry capable of measuring the low current of each channel and a high-voltage application circuit [4]. The plastic scintillators have the advantage of varying the size of the pixels, but they have the disadvantage of including light sensors for collecting the generated light $[5,6]$. On the other hand, the diode-based dosimetry device has a high reaction efficiency unlike the ion chamber used in the air state, and the $\mathrm{X}$-ray spatial distribution measuring device can be manufactured with a small pixel size and/or with a low cost diode base so it has an advantage in terms of price too. In addition, since it has high linearity with respect to the incident X-ray, it is suitable for the measurement of the spatial dose distribution for a wide range of $\mathrm{X}$-ray energy. However, the change of the leakage current due to the temperature and the increase of the cumulative dose caused by long time measurement may cause the degradation of the signal response performance $[4,7]$.

We developed a diode-based 128-channel X-ray spatial dose distribution measuring device for the measurement of the X-ray spatial dose distribution of container detectors among these various types of dose measurement methods and performed the measurement experiment in a container
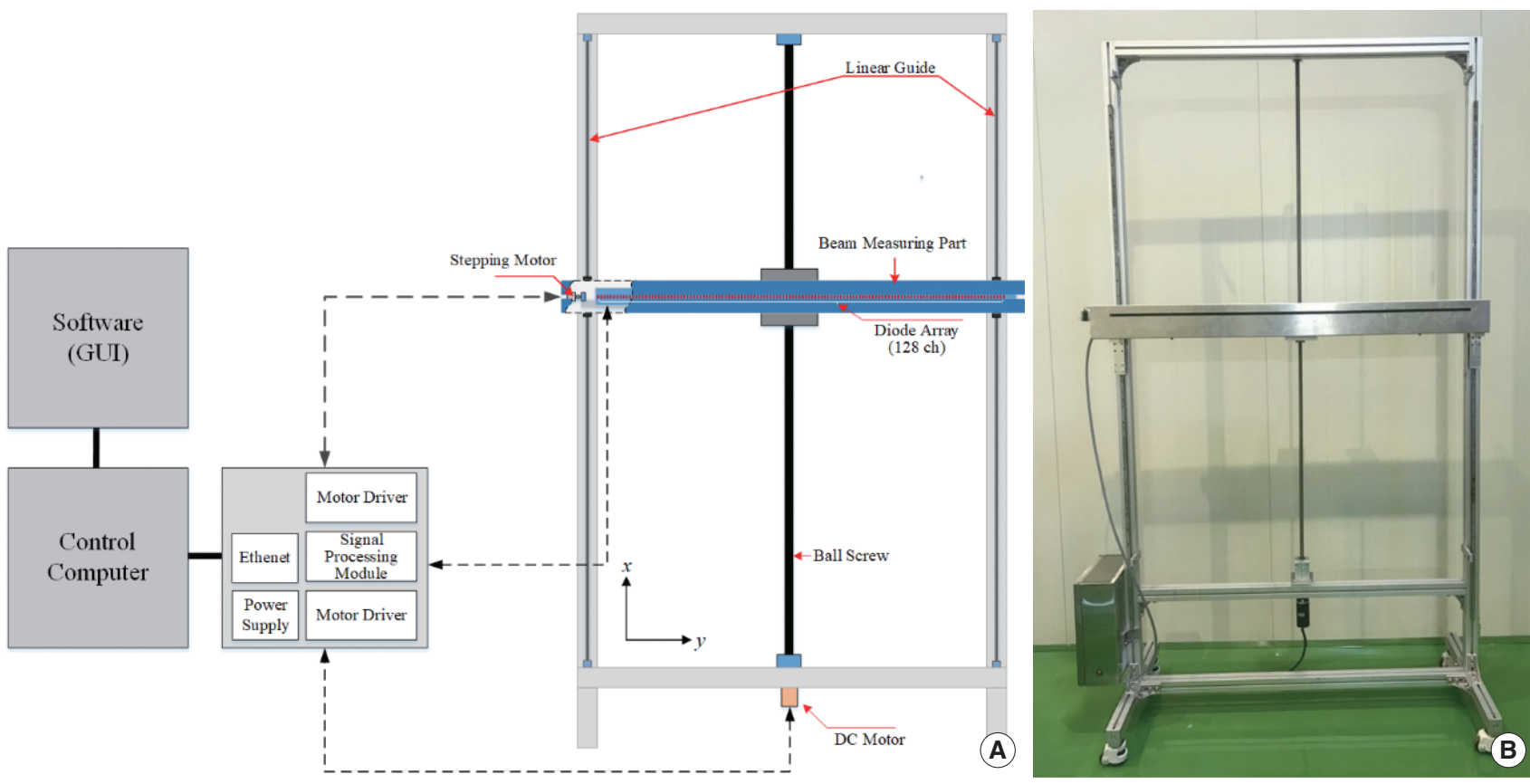

Fig. 1. The schematic diagram of X-ray spatial dose distribution measuring system (A) and real system (B); the developed X-ray spatial dose measuring device consists of a sensor part for dose measurement, a sensor movement part, and a signal processing part. 
detector using an X-ray generator with the energy of up to 9 $\mathrm{MeV}$ for the performance evaluation. The performance evaluation of the developed device included the measurements of the X-ray response characteristics by horizontal position, the linearity of response depending on X-ray incident amount, high resolution distribution, spatial distribution and others.

\section{Materials and Methods}

\section{X-ray spatial dose distribution measuring device}

As shown in Figure 1, the high-energy X-ray spatial dose distribution measuring device described in this paper consists of the X-ray sensitive sensor part for collecting incident $\mathrm{X}$-rays, the signal processing part for converting and transferring the signals collected by the sensor, and the sensor transfer part for automatically adjusting the position of the sensor part in the vertical and horizontal directions. Its maximum measurable range is $1,500 \mathrm{~mm}$ (vertical) $\times 1,280 \mathrm{~mm}$ (horizontal). In the system configuration, the X-ray sensitive sensor part has a horizontal array structure using $128 \mathrm{X}$-ray measurement diodes (SMLJ60S10, Micro Commercial Components, Dallas, TX) as X-ray sensitive sensors, and 64 channels of sensors are installed on a set of electronic circuit boards to constitute two sensor units in total as shown in
Figure 2A. In addition, each sensor is attached horizontally at intervals of $10 \mathrm{~mm}$ as shown in Figure 2B. The signal processing part is constructed by using a signal processing element (DDC264, Texas Instruments, Dallas, TX) equipped with 64 channels of dual current indicators and A/D converter (Figure 3). The X-ray incident on each sensor is measured by using a current integral form, which measures the voltage after accumulating the current generated by the response between the X-ray photons and the sensors in a capacitor for a certain period of time. In order to continuously collect the input charge, two integrators are attached to each channel so that one integrator can perform the A/D conversion and initialization of the output of the other integrator while the latter integrates. If the input current from the sensor part to the integrator is $I_{\text {in }}$ and the capacitance for the current accumulation of the integrator is $C_{\mathrm{f}}$, the output process of the measured charge quantity $Q_{0}$ for measurement is as follows. $I_{\text {in }}$ is accumulated in $C_{\mathrm{f}}$ by the output from an opamp, and the output voltage $V_{\mathrm{o}}$ is determined by the relationship between $I_{\text {in }}$ and $C_{\mathrm{f}}$. When the voltage/charge characteristic of $C_{\mathrm{f}}$ has a linear characteristic, the measured charge $Q_{\mathrm{o}}$ can be expressed as a product of $V_{\mathrm{o}}$ and $C_{\mathrm{f}}$, and $Q_{\mathrm{o}}$ can be expressed as an integral of $I_{\text {in }}$ with respect to time. Therefore, if $C_{\mathrm{f}}$ is initialized at the initial stage of integration and the current is accumulated for a certain time $(t)$, the total

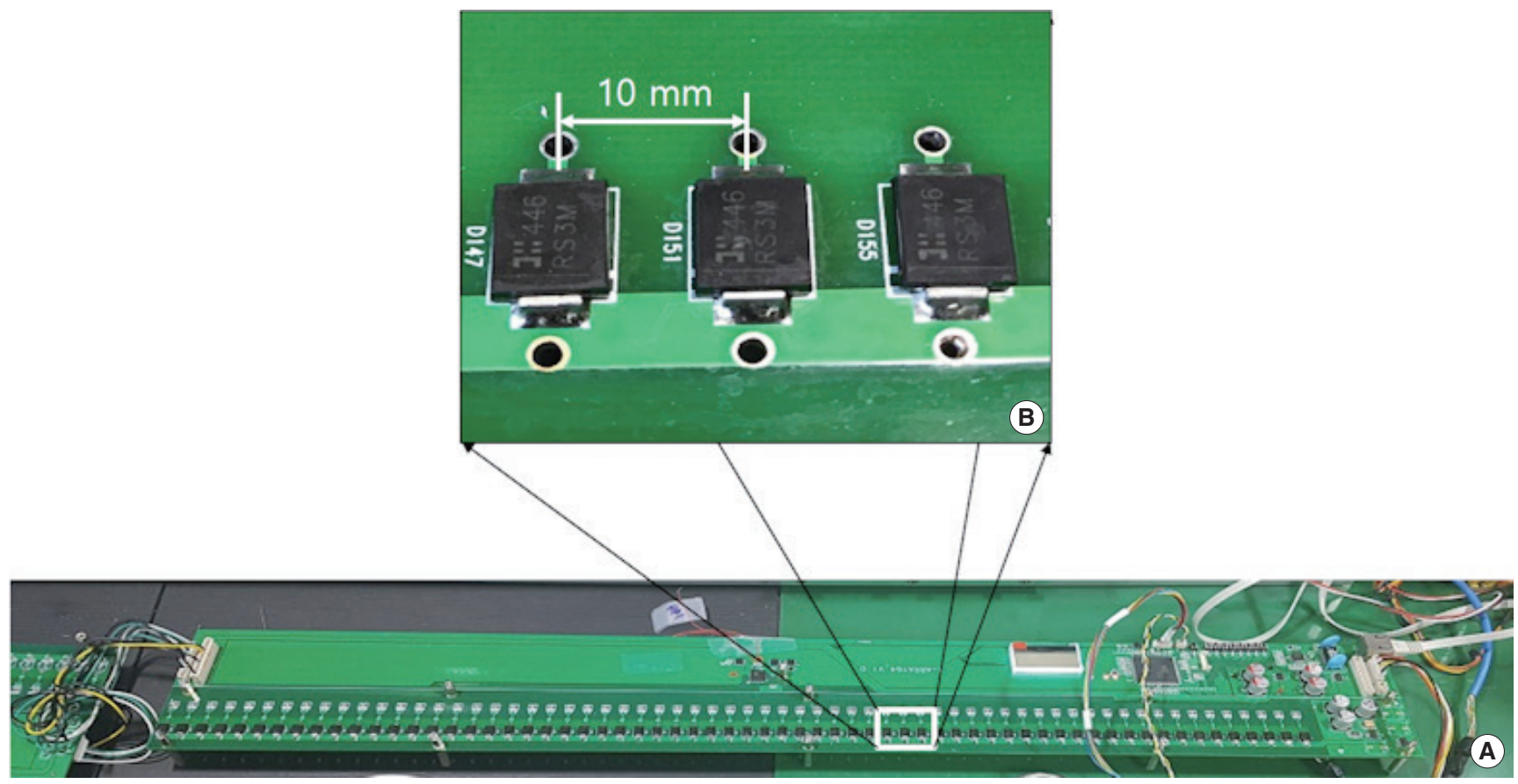

Fig. 2. The sensor part consisting of 68 diodes (A) and diode attached to the circuit board (B); the sensor part of 128 channels for X-ray dose measuring consists of two sets of 68 diode-based circuit boards and the pitch of each diode attached to the sensor part is $10 \mathrm{~mm}$. 


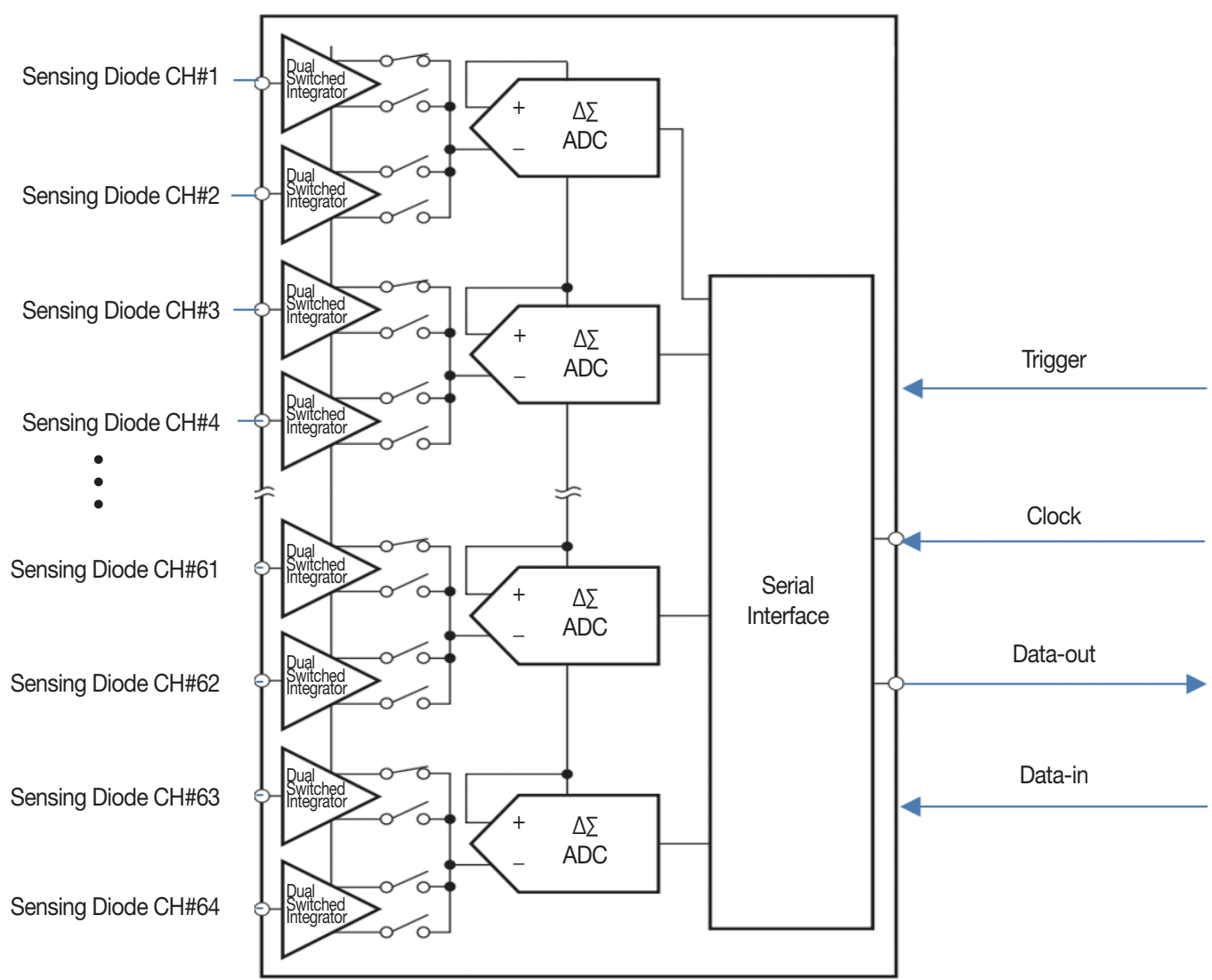

Fig. 3. A signal processing part for processing a signal output from the sensors; the signal transmitted from each sensing diode is transmitted and converted by a dual switched integrator and an analog-to-digital converter.

amount of the input charge during the corresponding time period can be obtained. Therefore, the quantity of electric charge can be expressed as;

$$
Q_{o}=V_{o} \times C_{f}=t \times I_{\text {in }}
$$

Using Equation 1, the input current (average value) can be calculated as;

$$
Q_{o}=V_{o} \times \frac{C_{f}}{t}
$$

As a result, the $C_{\mathrm{f}}$ of the integrator can be determined on the basis of the magnitude of the current generated by the sensor after the generated $\mathrm{X}$-ray photons reach the sensor for detection. Therefore, the magnitude of the generated current must be determined in order to determine $C_{\text {f }}$. The magnitude of the current is $I_{\text {in, }}$ which is generated by the sensor collecting X-rays, and $I_{\text {in }}$ can be calculated by using the X-ray dose generated by the X-ray generator and the X-ray collection efficiency of the diode. Based on the calculation of the current generated from the sensor, the value of $C_{\mathrm{f}}$ provided by the signal processing element is set to $37.5 \mathrm{pF}$, which can be set in the signal processing element. As a result, since the maximum value of $V_{0}$ is $4.096 \mathrm{~V}$, the maximum cumulative charge amount is $37.5 \mathrm{pF} \times 4.096 \mathrm{~V}=153.6 \mathrm{pC}$. In this system, the current accumulation time is set to $5 \mathrm{~ms}$ and, consequently, the maximum value of the input current is $153.6 \mathrm{pC}$ per $5 \mathrm{~ms}$. Since the magnitude of the current measured with the diode at a point $1 \mathrm{~m}$ away from the $\mathrm{X}$-ray generation source is approximately $13.1 \mathrm{nA}$, the maximum current generated by the measuring diode is $13 \mathrm{nA}$ or less when measuring the dose at a position more than $1 \mathrm{~m}$ away from the $\mathrm{X}$-ray source of the generator. The A/D conversion of the developed system is executed in 20 bits, and the processed signal is transmitted to the operation computer at intervals of $10 \mathrm{~ms}$.

As described above, the sensitive sensor part of the developed system consists of a 128-channel diode horizontally arranged at intervals of $10 \mathrm{~mm}$. In addition, the part can perform the measurement of the dose distribution for the area up to $1,500 \mathrm{~mm}$ in the vertical direction. To do this, the system measures the spatial dose after moving the sensor part in the vertical direction. As shown in Figure 1, a 2,000 mm 
ball screw and a driving DC motor is attached to the center of the system to transfer the sensor in the vertical direction. In order to help smooth vertical transfer of the sensor part, linear guides are attached to both ends of the sensor part. The vertical measurement interval can be adjusted from a minimum of $1 \mathrm{~mm}$ to a measurable range. In addition, the developed system was designed to horizontally move the sensor part from a minimum of $1 \mathrm{~mm}$ to a maximum of 10 $\mathrm{mm}$. Since the spacing between sensors is $10 \mathrm{~mm}$, the measurable horizontal spatial resolution cannot exceed $10 \mathrm{~mm}$. Therefore, in order to improve the horizontal spatial, the horizontal sensor transfer part composed of a small stepping motor and a ball screw is installed to the developed system so as to measure the dose after horizontally moving the sensor part (1-10 mm). The spatial dose measuring device developed with these functions can measure the spatial dose for an area of up to $1,500 \mathrm{~mm}$ (vertical) $\times 1,280 \mathrm{~mm}$ (horizontal) at intervals of maximum $1 \mathrm{~mm}$ in the vertical and horizontal directions.

\section{Evaluation of $\mathrm{X}$-ray response characteristics of distribution measuring device}

The evaluation of response characteristics of the developed X-ray spatial dose distribution measuring device is based on the comparison about the signal gain difference of each pixel at the maximum X-ray source energies of $6 \mathrm{MeV}$ and $9 \mathrm{MeV}$, the response linearity according to the X-ray incident dose change, and horizontal spatial resolution. The measurement experiment was carried out at the container detector facility (Korea Research Institute of Ships \& Ocean Engineering, Gwangyang, Korea). The X-ray generator of the container detector facility was Linatron Mi- $9^{\mathrm{TM}}$ from Varex ${ }^{\circledR}$, which was capable of generating $6 \mathrm{MVp}$ and $9 \mathrm{MVp}$ X-rays, and had the maximum X-ray generation dose of $30 \mathrm{~Gy} \cdot \mathrm{min}^{-1}$. The collimator in front of the generator was configured to ir-

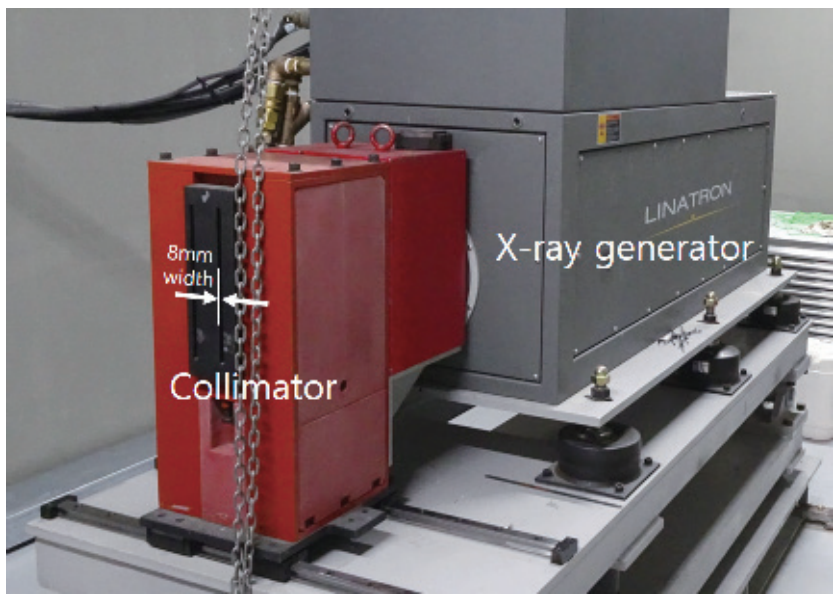

Fig. 4. LINAC and X-ray beam collimators for X-ray generation; the width of the collimator is $8 \mathrm{~mm}$.

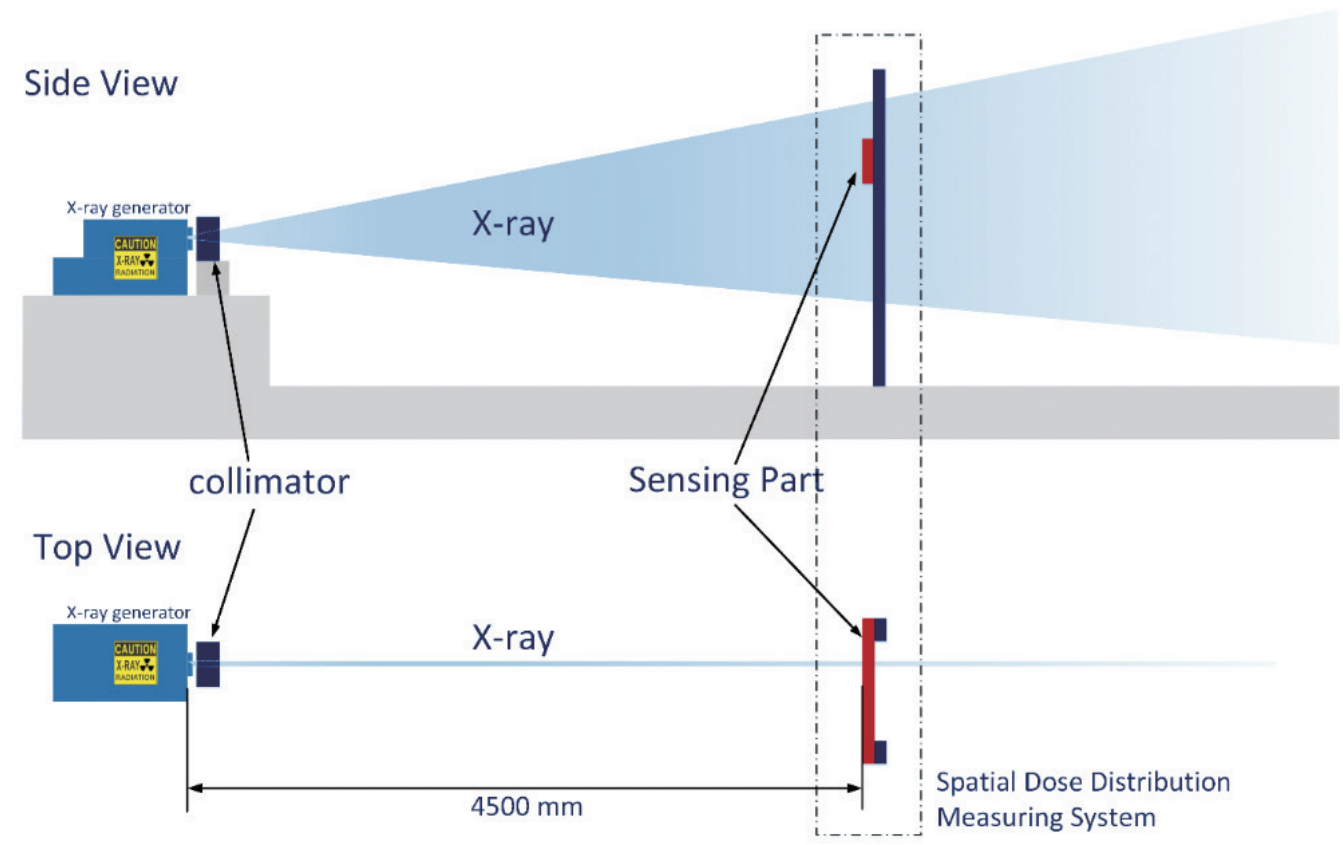

Fig. 5. Schematic diagram for performance evaluation experiment of X-ray spatial distribution measuring device; the distance between the $X$ ray generating position and the $\mathrm{X}$-ray spatial dose measuring device is $4,500 \mathrm{~mm}$. 


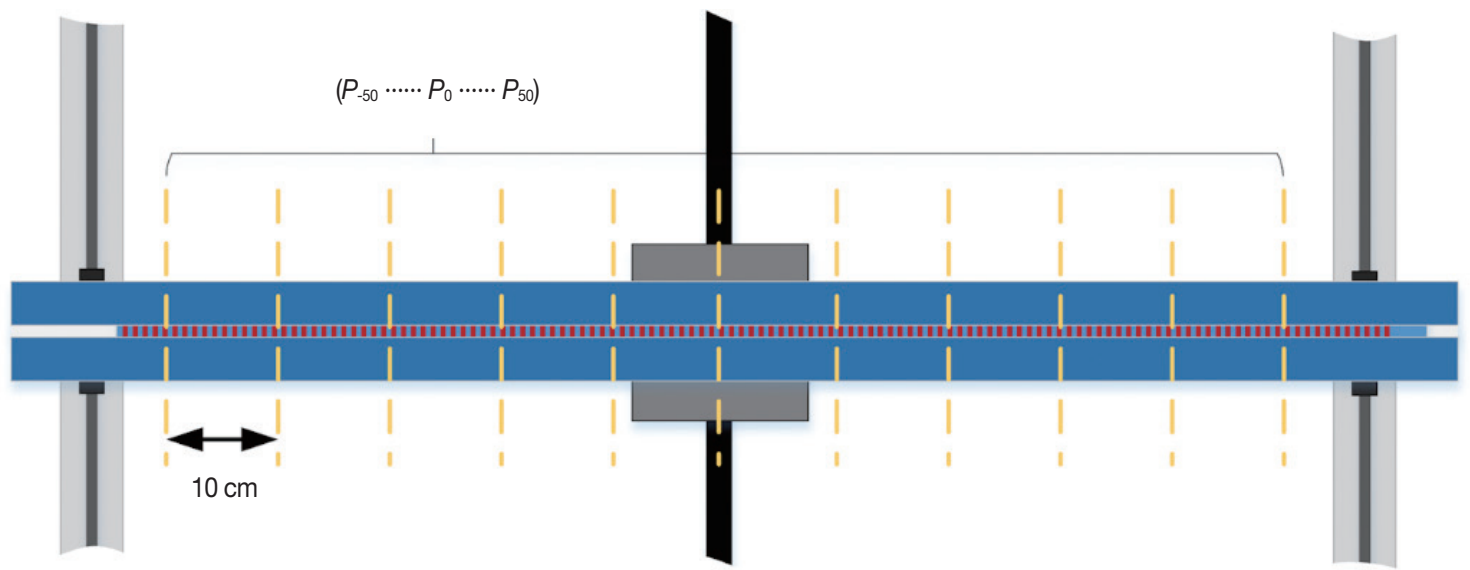

Fig. 6. X-ray measurement point for evaluating the response signal uniformity of the X-ray spatial distribution measuring device; measurement at 12 points (10 $\mathrm{cm}$ intervals).

radiate X-rays in the form of a fan-beam due to the nature of the research institute, and the width of the collimator was 8 $\mathrm{mm}$ (Figure 4). In order to evaluate the response characteristics of the X-ray generated by the developed system, the distance between the X-ray generator and the measuring device was set to $4.5 \mathrm{~m}$ as shown in Figure 5 .

First, the response characteristics depending on the X-ray generation dose were evaluated by varying the dose generated from the X-ray. The X-ray generation dose can be adjusted by changing the output pulse of the generator. In this study, the dose was adjusted by varying the $\mathrm{X}$-ray generation cycle of the generator within the range of 25 to $300 \mathrm{~Hz}$, and the change of the generated dose could be confirmed through the readings of the dosimeter attached to the X-ray generator. Second, the signal gain of each pixel was measured by changing the incident position of the X-ray at the intervals of $100 \mathrm{~mm}$ from the left to the right of the measuring device as shown in Figure 6, and then the response results from each pixel were compared. Third, for the high resolution spatial dose measurement experiment of the system, the responses to the signals were measured by moving the horizontal transfer device at intervals of $1 \mathrm{~mm}$. Finally, the spatial dose distributions from 750 to $1,200 \mathrm{~mm}$ relative to the ground were measured in the vertical direction in order to confirm the vertical and horizontal spatial measurement capability.

\section{Computer simulation of $\mathrm{X}$-ray spatial dose distribution measuring device}

The verification for the dose effectiveness of the developed $\mathrm{X}$-ray dose distribution measuring device was performed by computer simulation. In this study, MCNP6 (Version 1.0,
RSICC, Oak Ridge, TN, USA) was used for the computer simulation. The simulation of the source term for the computer simulation was generated by colliding electrons with the tungsten target as in the above-described $\mathrm{X}$-ray generation process of the linear accelerator for X-ray generation, and a collimator with the width of $8 \mathrm{~mm}$ was added to the computer simulation model in order to irradiate the generated X-ray in the form of fan beam. In addition, the computer simulation model of the detector for measuring dose distribution was composed of 129 cells in the horizontal direction with a rectangle of $10 \mathrm{~mm}$ (width) $\times 10 \mathrm{~mm}$ (length) $\times 1 \mathrm{~mm}$ (thickness). The response results were measured in each cell with *F8 tally. The results obtained by this method were converted into current values considering the reaction area ( $\left.V_{\text {active }}\right)$ of the photons by the actual diode, the ionization energy (3.6 $\mathrm{eV})$ and the number of electrons generated in the actual linear accelerator $\left(N_{\text {gen }}\right)$. Therefore, the current value $(I)$ in $\mathrm{n}$ channel can be calculated as;

$$
I(n)=\frac{E_{a b s}(n)}{3.6 \mathrm{eV}} \times N_{\text {gen }} \times V_{\text {active }} \times\left(1.6 \times 10^{-19}\right)
$$

\section{Results and Discussion}

\section{Uniformity}

As described above, the high-energy X-ray dose distribution measuring device developed consists of 128 sensors in the horizontal direction. Therefore, in order to accurately represent the spatial dose distribution, there should not be a big difference between signal response gain and noise of each sensor. In order to confirm the difference of the signal response gain according to the position of the developed 


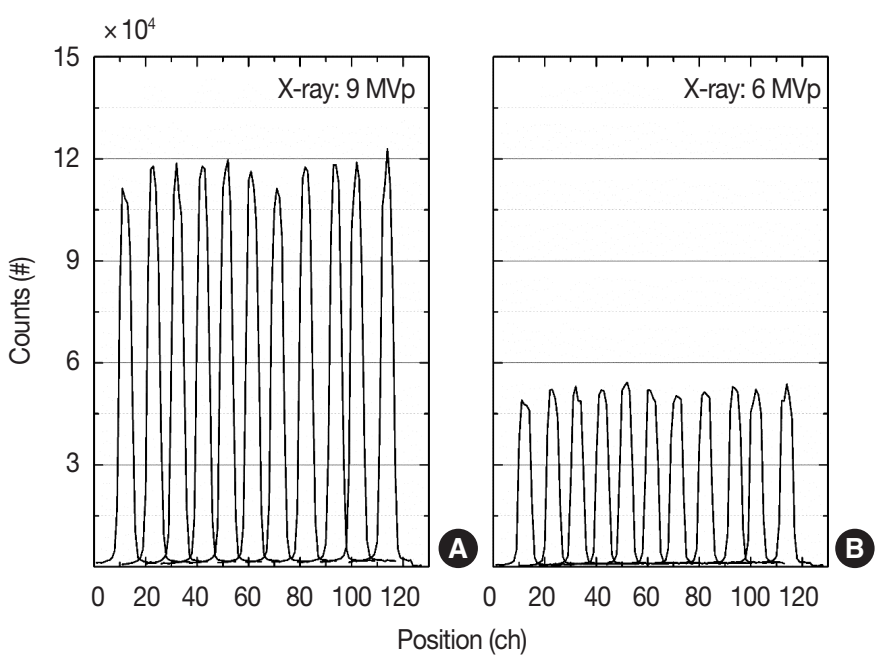

Fig. 7. Evaluation results of response signal uniformity of X-ray spatial distribution measuring device; 9MVp (A) and 6MVp (B).

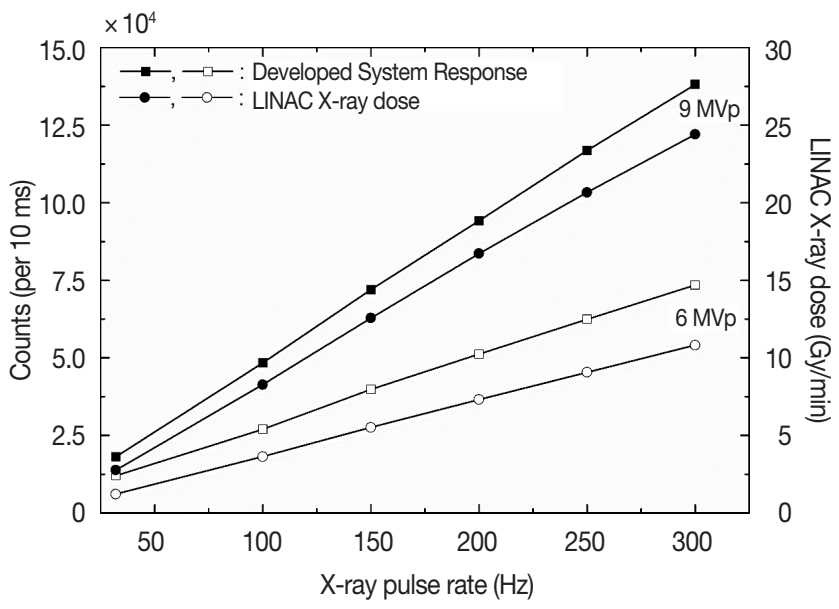

Fig. 8. The linearity of the response signal of the X-ray spatial distribution measuring device according to the dose change of the $\mathrm{X}$-ray generator.

system, $9 \mathrm{MVp}$ and $6 \mathrm{MVp}$ X-rays with the width of $8 \mathrm{~mm}$ were irradiated to the measuring device at a certain interval $(100 \mathrm{~mm})$ and then the response characteristics were evaluated. Figure 7 shows the response characteristics for each $\mathrm{X}$ ray incidence position. It can be seen that the difference between the maximum value and the minimum value of the response signals measured at each position of both $9 \mathrm{MVp}$ and $6 \mathrm{MVp}$ X-rays is about $10 \%$. Therefore, the uniformity of the response signal for each channel of the system will not have a significant effect on the measurement of the X-ray spatial dose distribution if the response signal is corrected considering the signal gain rate of each pixel.

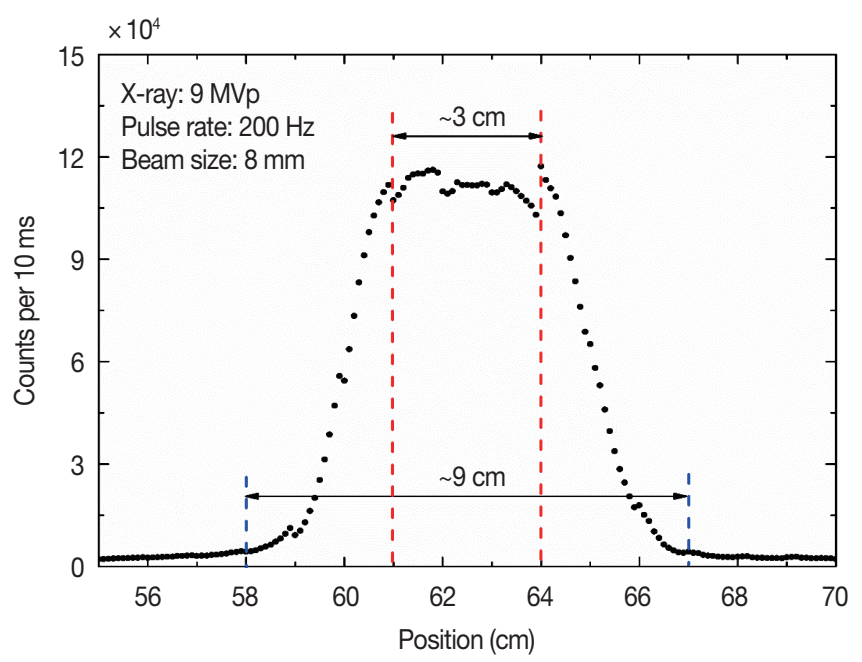

Fig. 9. Results of high-resolution measurement of X-ray spatial distribution measurement.

\section{Linearity}

In this study, the response characteristics were measured according to the X-ray generation dose in order to evaluate the linearity of the system response signal. Figure 8 shows the relationship between the response characteristics of the measuring device and the X-ray generation dose according to the change in the dose. It is confirmed that, in the case of the X-ray energies generated in both the $9 \mathrm{MVp}$ and the 6 MVp X-rays, the X-ray generation dose (circular symbol) measured by the internal dose measuring device in the generator and the change trend (square) of the response signal are similar and linearly increased. However, a close look at the dose change curve of the figure reveals a slight difference in the dose gradients measured by the internal dose measuring device and the developed device. This is considered to be attributed to the differences in X-ray response characteristics between the internal dose measuring device of the ion chamber based generator and the diode-based dose measuring device.

\section{Spatial resolution}

As described above, the developed system was designed to measure the dose while automatically moving the measuring device at intervals of $1 \mathrm{~mm}$ in order to measure the highresolution dose distribution. Figure 9 shows the results of high-resolution X-ray spatial distribution measurements. An $\mathrm{X}$-ray beam limited to a width of $8 \mathrm{~mm}$ was irradiated by an $\mathrm{X}$-ray beam collimator, but it can be seen that the signals were spread to the range of about $90 \mathrm{~mm}$, which included a flat portion of about $30 \mathrm{~mm}$ that the beam was directly irra- 
diated to plus and a portion gently measured due to the Xrays estimated by the scattered rays other than the direct Xrays. This is considered as a phenomenon caused by the distance between the collimator and the measuring device. As a result, it is estimated that the developed system can acquire a high-resolution spatial dose distribution. However, as shown in the figure, discontinuous data was confirmed in some sections. This phenomenon is considered to be caused by the fact that the signal gain of the measuring device has not been corrected. We plan to develop and improve the correction technique in the future.

\section{2-dimentional spatial distribution measurement}

Figure 10 shows the measurement results of the two-dimensional spatial distribution of incident X-rays. Figure 10A
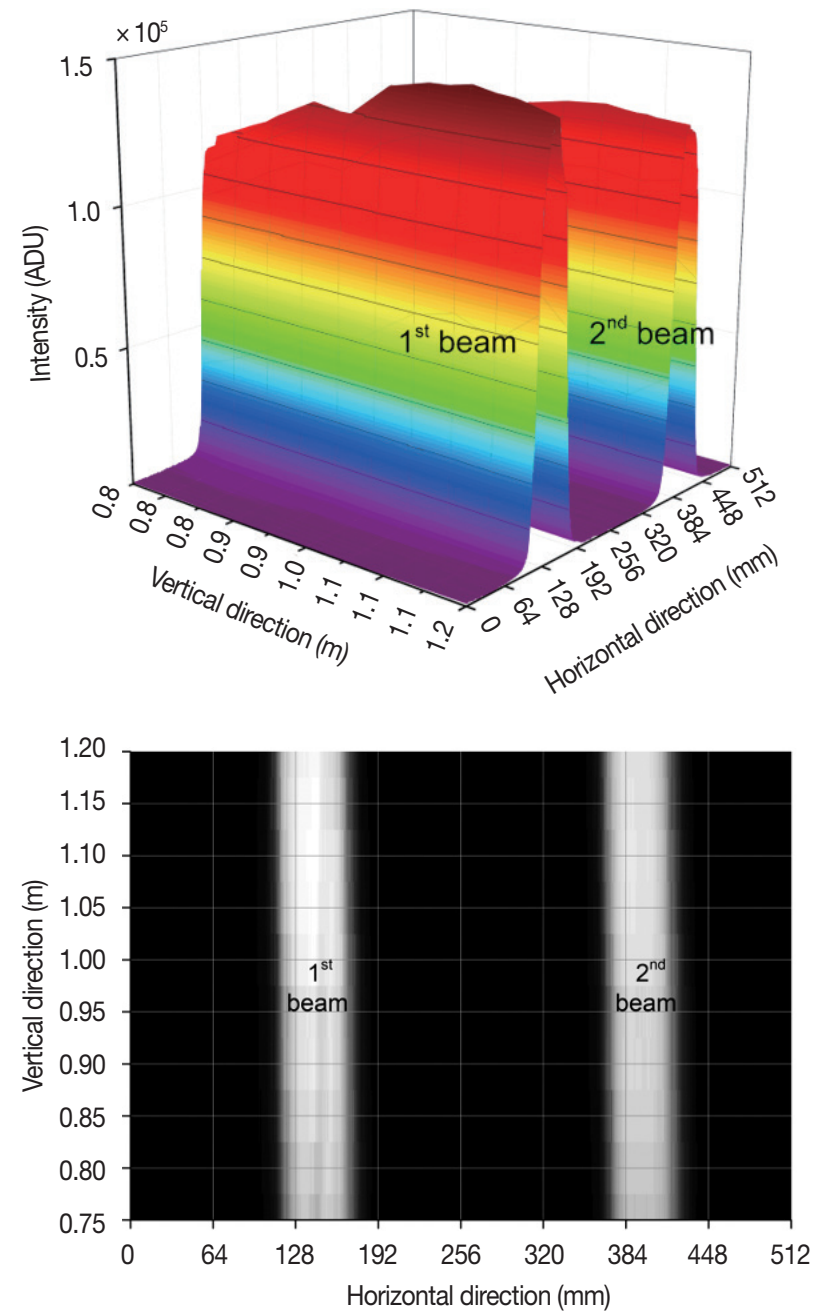

Fig. 10. The result of $X$-ray spatial distribution measurement; 3-dimensional graph of measurement results $(A)$ and a 2-dimensional graph (B). shows the results obtained in the two-dimensional space in a three-dimensional graph, and Figure 10B shows them in two dimensions. The measuring device shows the spatial doses for the doses irradiated from the first beam and the second beam because the beam travel path of the collimator located at the front of the X-ray generator is divided into two parts (Figure 3). Therefore, the X-ray generator of this container scanning equipment generates X-rays in the form of two fan beams (width $8 \mathrm{~mm}$ ). The two-dimensional spatial distribution was measured between 800 and 1,200 $\mathrm{mm}$ at intervals of $100 \mathrm{~mm}$ in the vertical direction and at intervals of 1 $\mathrm{mm}$ intervals in the horizontal direction. In Figure 10A, it can be seen that the magnitude of the dose measured increases as the vertical position direction changes, and the response signal decreases at around 1,100 mm. In addition, Figure 10B shows that the width of the incident beam is uniform.

\section{Effectiveness evaluation of $X$-ray spatial dose measuring device}

Figure 11 shows the results of computer simulation using MCNP and the measurement results using the spatial dose measuring device. The graph shows relative values to compare the two results and the distribution obtained from the experiment is relatively narrow compared with the computer simulation results. These results are considered to be attributed to two causes. The first case occurs when the collimator in front of the generator is not aligned with the source of the $\mathrm{X}$-ray generator. In such case, the width of the collimator may become narrower. The second case may occur when the focal spot of the X-ray source term in computer simulation is

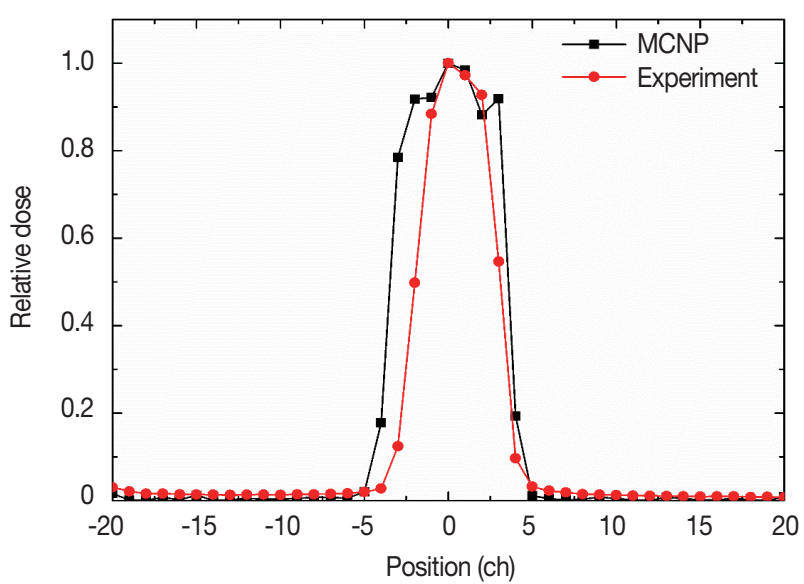

Fig. 11. The result of X-ray spatial distribution measurement; simulation results (square symbol) and measured result (circular symbol). 
different from the actual generator. Consequently, it can be seen that more precise experiments are required, but the difference between the results of the experiment and the computer simulation is not significant.

\section{Conclusion}

In the container detector facility, the X-ray spatial dose measuring device is necessary for the improvement of image quality and the optimization of a shielding structure as mentioned in the introduction. This paper describes the development of the X-ray spatial dose measuring device and the evaluation results about linearity of response signals, spatial uniformity, high resolution measurement ability, and spatial dose measurement ability using the developed device. As describe in this paper, it was confirmed that the system developed could clearly measure the spatial doses of X-rays, and the response characteristics of incident $\mathrm{X}$-rays of the system itself were also linear. In addition, it was confirmed that the response characteristics according to the position of incident X-rays were not bad as about $10 \%$ in relative error. However, since all the response characteristics for each of the 128 sensors have not been measured, it is considered that more precise evaluation on the additional system and further correction for the response gain of each sensor are required in the future. We will carry out the measurement of the total spatial dose of the container detector constructed by using the developed 2D spatial dose measuring device.

\section{Acknowledgements}

This research was supported by a grant from National R\&D Project of "Research on Fundamental Core Technology for Ubiquitous Shipping and Logistics" funded by Korea Institute of Marine Science and Technology Promotion (PMS3791).

\section{References}

1. Zentai G. X-ray imaging for homeland security. Int. J. Signal and Imaging Syst. Eng. 2010;3(1):13-20.

2. Chen G. Understanding X-ray cargo imaging. Nucl. Instrum. Methods Phys. Res., Sect. B. 2005;241(1-4):810-815.

3. Cho Y, Moon JH, Kang CS. Analysis of activation products generated from photoneutron capture reactions inside a linear accelerator used in a cargo inspection facility. Nucl. Instrum. Methods Phys. Res., Sect. B. 2007;265(2):615-620.

4. Low DA, Moran JM, Dempsey JF, Dong L, Oldham M, Dosimetry tools and techniques for IMRT. Med. Phys. 2011;38(3):13131338.

5. Perera H, Williamson JF, Monthofer P, Binns WR, Klarmann J, Fuller GL, Wong JW. Rapid two-dimensional dose measurement in brachytherapy using plastic scintillator sheet: linearity, signalto-noise ratio, and energy response characteristics. Int. J. Radiat. Oncol. Biol. Phys. 1992;23(5):1059-1069.

6. Beddar AS. Plastic scintillation dosimetry and its application to radiotherapy. Radiat. Meas. 2006;41:S124-S133.

7. Dixon RL, Ekstrand KE. Silicon diode dosimetry. Int. J. Appl. Radiat. Isot. 1982;33(11):1171-1176. 


\section{Appendix A. Diode based sensor}

As described in this paper, the X-ray to be measured in this study is generated by Linatron Mi-9 from VAREX company, which is capable of generating 6 $\mathrm{MVp}$ and $9 \mathrm{MVp}$ X-rays and has the maximum X-ray dose of $30 \mathrm{~Gy} \cdot \mathrm{min}^{-1}\left(10 \times 10 \mathrm{~cm}^{2}\right)$. The dose generated can be converted as follows;

$$
30 \mathrm{~Gy} \cdot \mathrm{min}^{-1}=0.5 \mathrm{~Gy} \cdot \mathrm{s}^{-1}=0.5 \mathrm{~J} \cdot(\mathrm{kg} \cdot \mathrm{s})^{-1}
$$

If the thickness of the diode is approximately $1 \mathrm{~mm}$, the efficiency with which $9 \mathrm{MVp}$ X-rays are collected by the diode is approximately $1.44 \%$. Given that the constituent material of the diode is silicon with a density of $2.33 \mathrm{~g} \cdot \mathrm{cm}^{-3}$, the energy delivered to the sensor for X-ray detection can be calculated as follows;

$$
0.5 \mathrm{~J} \cdot(\mathrm{kg} \cdot \mathrm{s})^{-1} \times 2.33 \mathrm{~g} \cdot \mathrm{cm}^{-3} \times 0.0144=1.68 \times 10^{-5} \mathrm{~J} \cdot \mathrm{cm}^{-3} \cdot \mathrm{s}^{-1}
$$

Since $1 \mathrm{eV}$ is $1.602 \times 10^{-19} \mathrm{~J}$, the result of Equation A-2 can be converted into electron volts as follows;

$$
1.68 \times 10^{-5} \mathrm{~J} \cdot \mathrm{s}^{-1}=1.04889 \times 10^{14} \mathrm{eV} \cdot \mathrm{cm}^{-3} \cdot \mathrm{s}^{-1}
$$

Since the size of the diode for X-ray measurement is $0.56 \mathrm{~cm}$ (width) $\times 0.66 \mathrm{~cm}$ (length) $\times 0.2 \mathrm{~cm}$ (thickness), the energy for the X-ray reaction area in the diode with $0.28 \mathrm{~cm}$ (width) $\times 0.1 \mathrm{~cm}$ (length) $\times 0.1 \mathrm{~cm}$ (thickness) can be calculated as;

$$
1.05 \times 10^{17} \mathrm{eV} \cdot \mathrm{cm}^{-3} \cdot \mathrm{s}^{-1} \times(0.28 \times 0.1 \times 0.1) \mathrm{cm}^{3}=2.94 \times 10^{11} \mathrm{eV} \cdot \mathrm{s}^{-1}
$$

Since the ionization energy of silicon is $3.6 \mathrm{eV}$, Equation A-4 can be converted as follows;

$$
\frac{2.94 \times 10^{12} \mathrm{eV} \cdot \mathrm{s}^{-1}}{3.6 \mathrm{eV}}=8.16 \times 10^{10} \mathrm{e}^{-} \cdot \mathrm{s}^{-1}
$$

Finally, since the charge amount of an electron is $1.6 \times 10^{-19} \mathrm{C}$, the generated current can be calculated as;

$$
\left(8.16 \times 10^{10}\right) \times\left(1.6 \times 10^{-19} C\right) \cdot s^{-1}=1.31 \times 10^{-8} A=13.1 n A
$$

